\title{
Dynamic Fuzzy Reliability Analysis of Multistate Systems Based on Universal Generating Function
}

\author{
Peng Gao $\mathbb{D}^{1,2}$ Liyang Xie, ${ }^{3}$ Wei Hu, ${ }^{4}$ Chang Liu, ${ }^{1}$ and Jinzhao Feng ${ }^{1}$ \\ ${ }^{1}$ School of Mechanical Engineering, Liaoning Shihua University, Liaoning 113001, China \\ ${ }^{2}$ School of Mechanical Engineering and Automation, Zhejiang Sci-Tech University, Hangzhou 310018, China \\ ${ }^{3}$ School of Mechanical Engineering and Automation, Northeastern University, Liaoning 110819, China \\ ${ }^{4}$ Department of Industrial Design, Liaoning Shihua University, Liaoning 113001, China
}

Correspondence should be addressed to Peng Gao; gaopeng@lnpu.edu.cn

Received 24 May 2017; Revised 22 March 2018; Accepted 4 April 2018; Published 10 May 2018

Academic Editor: Gen Q. Xu

Copyright (c) 2018 Peng Gao et al. This is an open access article distributed under the Creative Commons Attribution License, which permits unrestricted use, distribution, and reproduction in any medium, provided the original work is properly cited.

\begin{abstract}
Considering the fuzziness of load, strength, operational states, and state probability, reliability models of multistate systems are developed based on universal generating function (UGF). The fuzzy UGF of load and the fuzzy UGF of strength are proposed in this paper, which are used to derive the fuzzy component UGF and the fuzzy system UGF. By defining the decomposition operator and the inner product operator, failure dependence and effects of multiple load applications are taken into account in the established reliability models. Moreover, dynamic fuzzy reliability models of multistate systems are constructed considering the strength degradation of components. The results show that failure dependence and the effects of multiple load applications have significant impacts on system reliability, which considerably decrease system reliability and increase the fuzziness of system reliability under low performance requirements. Besides, in the dynamic reliability analysis of multistate systems, strength degradation dependence could lead to large computational error.
\end{abstract}

\section{Introduction}

Reliability is an important index in quality evaluation [1]. Traditional hypothesis of binary state about technological systems cannot always be satisfied in practice. Partial failure or degradation of systems could be encountered. In this case, it is insufficient to define the states of the systems by working normally or failing to work. Thus, reliability analysis of multistate systems has become more and more attractive. The universal generating function (UGF) is important and effective mathematical means to calculate the reliability of multistate systems. Compared with other methods, the UGF can clearly present the mapping relation between the working states of the systems or the components and the corresponding state probabilities. Besides, high computational efficiency is another important advantage of the UGF. Hence, the UGF has been widely used in reliability analysis of multistate systems such as electric power systems, electronic systems, and mechanical systems.
In the last few decades, a great deal of innovative research has been carried out on the reliability analysis of multistate systems based the UGF technology. For instance, a new expression was proposed for the UGF counting (nonsingular) walks with small steps in the quarter plane in terms of infinite series by Kurkova and Raschel [2]. Besides, three cases (an algebraic case, a transcendental D-finite case, and an infinite group model) are used to illustrate the expression of the generating function in the literature. Dherin proved that this UGF converges for analytical Poisson structures and shows that the induced local symplectic groupoid coincides with the phase space of Karasev-Maslov [3]. The method for probabilistic production simulation of wind power systems was developed based on universal generating function (UGF) by Jin et al. which completes the production simulation with the chronological wind power and load demand [4]. Farsi developed a new method for reliability evaluation via the UGF for a solar array mechanism [5]. Levitin provided a detailed discussion on the origin and the algorithm of UGF [6]. 


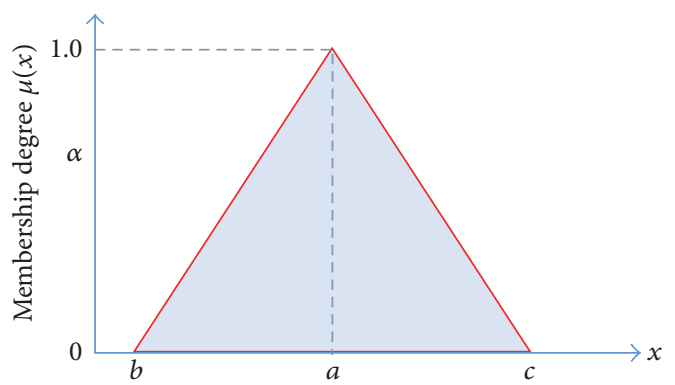

FIgURE 1: Membership degree of a triangular fuzzy number.

Moreover, a series of reliability models were given for various typical systems (such as series system, parallel system, and voting system), which laid the foundation for reliability analysis of multistate systems by using the method of UGF. In addition, the problem of reliability-based optimization of multistate system can be addressed by combining the UGF with various optimization algorithms such as the genetic algorithm, and ant colony algorithm. [7, 8]. Ding and Lisnianski proposed the fuzzy UGF technique to analyze the reliability of a series-parallel system [9].

For many technology systems, the system states are closely related to the working load $[10,11]$. Meanwhile, the failure of these systems always occurs in the case where the strength exceeds the working load. Further investigation of load effects on multistate systems should be carried out. In addition, to explore the randomness of the variables in the UGF of multistate systems and components, a large amount of samples is required. In some situations, samples are insufficient, some uncertain information is based on experience and judgment, or experimental data is obtained under the unknown or nonconstant reproduction conditions [12-15]. In this case, fuzzy mathematics theory can be used as an alternative method to analyze the system reliability [1618]. In practice, state degradation is always encountered, such as the degradation in the failure modes of fatigue, abrasion, or corrosion, which makes the system states different at different time instants. The system states and the corresponding possibility that these states show obvious dynamic characteristics. In order to describe the change of system states, state possibility and system reliability with time and dynamic fuzzy reliability models of multistate systems are developed in this paper. Moreover, in the models, the factors of both load and strength are taken into consideration in component reliability derivation, which facilitates the quantitative description of the influences of the failure dependence on system reliability.

\section{Load UGF and Strength UGF}

As a matter of fact, it is important to obtain the fuzzy component states and corresponding fuzzy probabilities. The concept of the component states and the corresponding probabilities in this paper are similar to that defined in $[6,10]$. However, the uncertainty in the component states and the corresponding probabilities at each load application is described by the membership degree. The detailed method to obtain the membership degree of a fuzzy variable can be referred to in [19].

The UGF establishes the mapping relationship between the states of the components or the systems and the occurrence probability of the corresponding states in the form of polynomial. Assume that the $i$ th $(i=1,2, \ldots, n)$ component in a system has $m_{i}$ states and the system has $m_{s}$ states. $g_{i j_{i}}$ and $p_{i j_{i}}$ represent the $j_{i}$ th state of the $i$ th $(i=1,2, \ldots, n)$ component and the corresponding probability, respectively. $g_{s j}$ and $p_{s j}$ are the state of the $s j$ th state of the system and the corresponding probability, respectively. Then the UGF $U_{i}$ of the $i$ th component and the UGF $U_{s}$ of the system can be expressed as

$$
\begin{gathered}
U_{i}=\sum_{j_{i}=1}^{m_{i}} p_{i j_{i}} z^{g_{i j_{i}}}, \\
U_{s}=\sum_{s j=1}^{m_{s}} p_{s j} z^{g_{s j}} .
\end{gathered}
$$

The operation between the UGF is carried out via different operators as follows:

$$
\begin{aligned}
\bigotimes_{f} & \left(U_{1}, U_{2}, \ldots, U_{n}\right) \\
= & \sum_{j_{1}=1}^{m_{1}} \sum_{j_{2}=1}^{m_{2}} \cdots \sum_{j_{n}=1}^{m_{n}} \prod_{i=1}^{n} p_{i j_{i}} z^{f\left(g_{1 j_{1}}, g_{2 j_{2}}, \ldots, g_{n j_{n}}\right)} .
\end{aligned}
$$

As mentioned above, we will be mainly concentrated on the fuzzy reliability of multistate systems in this paper. The fuzzy UGF can be expressed as [9]

$$
\widetilde{U}=\sum_{i=1}^{k} \widetilde{p}_{i} z^{\widetilde{g}_{i}}
$$

where $\widetilde{p}_{i}, \widetilde{g}_{i}$ stand for the fuzzy state probability and fuzzy working state, respectively. For computational convenience, the triangular fuzzy number is always used to model the fuzzy variables in reliability models. The triangular fuzzy number can be expressed as $(a, b, c)$ with the membership function shown in Figure 1 and

$$
\mu_{X}(x)= \begin{cases}0, & x<b, \\ \frac{x-b}{b-a}, & b<x<a \\ \frac{c-x}{c-a}, & a<x<c \\ 0, & x>c .\end{cases}
$$

The computation rule between the triangular fuzzy numbers can be referred to in [15]. When $\widetilde{p}_{i}, \widetilde{g}_{i}$ are deterministic value, the fuzzy UGF becomes the traditional UGF. To derive 
the fuzzy UGF of the components and systems, the fuzzy UGFs of load and strength are defined as follows:

$$
\begin{gathered}
\widetilde{S}=\sum_{i=1}^{n_{1}} \widetilde{p}_{i} z^{\widetilde{s}_{i}}, \\
\widetilde{H}=\sum_{j=1}^{n_{2}} \widetilde{p}_{j} z^{\widetilde{r}_{j}},
\end{gathered}
$$

where $\widetilde{s}_{i}, \widetilde{r}_{j}, \widetilde{p}_{i}, \widetilde{p}_{j}$ can be deterministic value or fuzzy numbers and represent discrete value of load, discrete value of strength, fuzzy probability of load, and fuzzy probability of strength, respectively. It should be noted that the strength mentioned in this paper refers to generalized strength. For instance, it can be residual strength in the failure mode of fatigue, heat resistance in system thermal analysis, or corrosion resistance in the failure mode of corrosion. Furthermore, under the continuous application of working load, strength could degrade with the existence of cavities, cracks in material of components. In this case, the strength UGF should be a function of time.

\section{Component UGF}

The states of components and systems are always defined or determined by the working load in power systems, electronic system, or mechanical systems. Thus, provided that the load UGF and the strength UGF are given, the component UGF can be derived as follows:

$$
U=\bigotimes_{f_{1}}(S, H)=\sum_{i=1}^{n_{1}} \sum_{j=1}^{n_{2}} \widetilde{p}_{i} \tilde{p}_{j} z^{f\left(s_{i}, r_{j}, g_{i}\right)},
$$

where $\widetilde{p}_{i} \widetilde{p}_{j}$ is the multiplication of fuzzy numbers, $g_{i}$ is the component state corresponding to $s_{i}$, and $f_{1}=1\left(s_{i}<r_{j}\right) \times g_{i}$ that will be explained in detail in the following section.

In this section, models of component UGFs are derived in different situations. Models in Section 3.1 are used in the case where the load is a constant and the strength is a fuzzy variable. Models in Section 3.2 apply to the situation where the strength is a constant and the load is a fuzzy variable. Models in Section 3.3 are for the calculation of component UGFs with the load and the strength both being fuzzy variables. Models in Section 3.4 are used to calculate component UGFs considering load application times. The models in Sections 3.1, 3.2, 3.3, and 3.4 do not take strength degradation into account. The models in Section 3.5 consider strength degradation.

\subsection{Component UGF with Deterministic Load and Fuzzy}

Strength. When the discrete value of the load is deterministic and the discrete value of the strength is a fuzzy number, denote the $j$ th discrete value of strength by $\left(a_{j}, b_{j}, c_{j}\right)$ and the $i$ th discrete value of load by $s_{i}$. The component UGF can be calculated as follows:

$$
U=\bigotimes_{f_{2}}(S, H)=\sum_{i=1}^{n_{1}} \sum_{j=1}^{n_{2}} \tilde{p}_{i} \tilde{p}_{j}\left[p_{i j} z^{g_{i}}+\left(1-p_{i j}\right) z^{0}\right],
$$

where $p_{i j}$ is calculated based on the equivalent probability density function method by [15]

$$
p_{i j}= \begin{cases}1, & s_{i}<b_{j}, \\ 1-\frac{\left(s_{i}-b_{j}\right)^{2}}{\left(c_{j}-b_{j}\right)\left(a_{j}-b_{j}\right)}, & b_{j}<s_{i}<a_{j}, \\ \frac{\left(c_{j}-s_{i}\right)^{2}}{\left(c_{j}-b_{j}\right)\left(c_{j}-a_{j}\right)}, & a_{j}<s_{i}<c_{j}, \\ 0, & s_{i}>c_{j} .\end{cases}
$$

3.2. Component UGF with Deterministic Strength and Fuzzy Load. When the discrete value of the load is fuzzy number and the discrete value of the strength is deterministic, denote $i$ th discrete value of the load by $\left(a_{i}, b_{i}, c_{i}\right)$ and the $j$ th discrete value of strength by $r_{j}$. The component UGF can be calculated as follows:

$$
U=\bigotimes_{f_{3}}(S, H)=\sum_{i=1}^{n_{1}} \sum_{j=1}^{n_{2}} \tilde{p}_{i} \tilde{p}_{j}\left[p_{i j} z^{g_{i}}+\left(1-p_{i j}\right) z^{0}\right],
$$

where $p_{i j}$ can be calculated by

$$
p_{i j}= \begin{cases}0, & r_{j}<b_{i}, \\ \frac{\left(r_{j}-b_{i}\right)^{2}}{\left(a_{i}-b_{i}\right)\left(c_{i}-b_{i}\right)}, & b_{i}<r_{j}<a_{i}, \\ 1-\frac{\left(c_{i}-r_{j}\right)^{2}}{\left(c_{i}-a_{i}\right)\left(c_{i}-b_{i}\right)}, & a_{i}<r_{j}<c_{i}, \\ 1, & r_{j}>c_{i} .\end{cases}
$$

3.3. Component UGF with Fuzzy Strength and Fuzzy Load. In the situation where the discrete value of the load and the discrete value of the strength are both fuzzy numbers, denote ith discrete value of load by $\left(a_{i}, b_{i}, c_{i}\right)$ with the membership function of $\mu_{S}(s)$ and the $j$ th discrete value of strength by $\left(a_{j}, b_{j}, c_{j}\right)$ with the membership function of $\mu_{H}(h)$. The component UGF can be calculated as follows:

$$
U=\bigotimes_{f_{4}}(S, H)=\sum_{i=1}^{n_{1}} \sum_{j=1}^{n_{2}} \tilde{p}_{i} \tilde{p}_{j}\left[p_{i j} z^{g_{i}}+\left(1-p_{i j}\right) z^{0}\right],
$$

where $p_{i j}$ can be given by

$$
\begin{aligned}
& p_{i j} \\
& \quad \begin{cases}0, & c_{j}<b_{i}, \\
\int_{b_{i}}^{c_{i}} \frac{2 \mu_{S}(s)}{\left(c_{i}-b_{i}\right)} d s \int_{s}^{c_{j}} \frac{2 \mu_{H}(h)}{\left(c_{j}-b_{j}\right)} d h, & b_{i}<c_{j}, b_{j}<c_{i}, \\
1, & c_{i}<b_{j} .\end{cases}
\end{aligned}
$$

3.4. Component UGF with Fuzzy Strength and Fuzzy Load Considering Load Application Times. Component UGF is the basis of the fuzzy reliability calculation of the multistate 


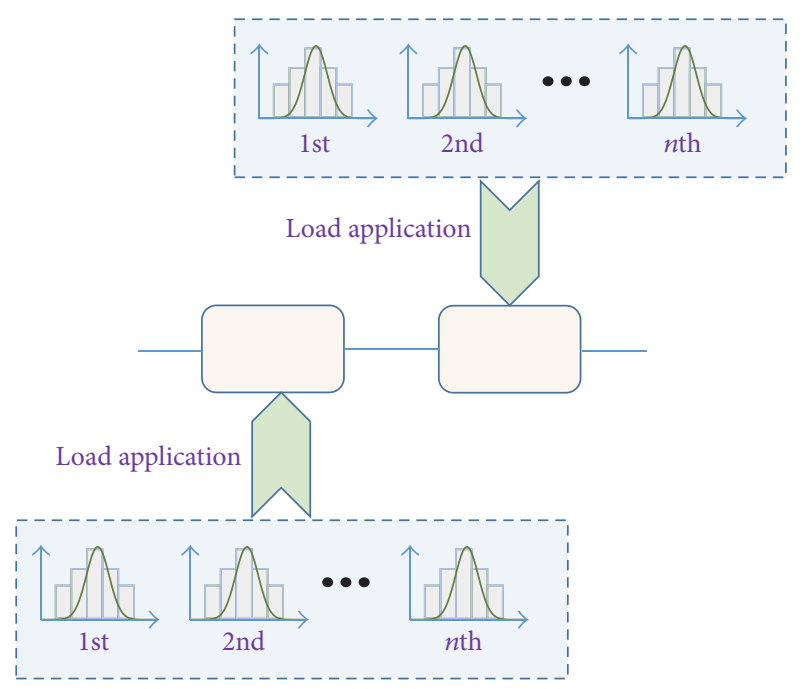

FIGURE 2: Components under multiple load applications.

system. The above analysis only considers the situation where load applies once and the effects of multiple loading application on component UGF are not taken into consideration. After the load application for $n$ times as shown in Figure 2, considering the expression of load UGF in (1), the maximum load UGF can be expressed as

$$
\begin{aligned}
U_{\widetilde{s} \max } & =\bigotimes_{\max }(\underbrace{\widetilde{S}, \widetilde{S}, \ldots, \widetilde{S}}_{n}) \\
& =\sum_{i_{1}=1}^{n_{1}} \sum_{i_{2}=1}^{n_{1}} \ldots \sum_{i_{n}=1}^{n_{1}} \prod_{j=1}^{n} \tilde{p}_{i_{j}} z^{\max \left(\widetilde{s}_{i_{1}}, \widetilde{s}_{i_{2}}, \ldots, \widetilde{s}_{i_{n}}\right)} .
\end{aligned}
$$

The operations in (14) follow the computational rule of fuzzy numbers. Substitute (14) into (7) and then the component UGF considers the effects of multiple loading application.

3.5. Component UGF Considering Strength Degradation. In practice, strength degradation of components is always encountered due to fatigue, wear, corrosion, and so on. When considering the strength degradation of components, conventional static UGF has to be extended to time-dependent UGF. In this section, we will be focused on gradual strength degradation. The operational duration $T$ of components is divided into a series of time intervals in which the component strength is assumed to be constant. The strength in the $k$ th time interval is denoted by

$$
r\left(t_{k}\right)=r_{0} \Phi\left(t_{k}\right)
$$

where $r_{0}$ is the initial strength. In the case where $r_{0}$ is a fuzzy number denoted by $(b, a, c)$, we only consider the degradation of center point $a$ of the fuzzy number with the both widths of $(a-b)$ and $(c-a)$ keeping constant. For a specified initial discrete value of strength $r_{0}$, the component UGF in the $k$ th time interval can be expressed as

$$
U_{1}\left(t_{k}\right)=\bigotimes_{f_{1}}\left[S, H\left(t_{k}\right)\right]=\sum_{i=1}^{n_{1}} \widetilde{p}_{i} z^{f\left(s_{i}, r\left(t_{k}\right), g_{i}\right)}
$$

Considering all the possible discrete value of initial strength, the time-dependent component UGF in the $k$ th time interval can be given by

$$
\begin{aligned}
U\left(t_{k}\right) & =\sum_{j=1}^{n_{2}} \tilde{p}_{j}\left\{\bigotimes_{f_{1}}\left[S, H\left(t_{k}\right)\right]\right\} \\
& =\sum_{j=1}^{n_{2}} \tilde{p}_{j}\left(\sum_{i=1}^{n_{1}} \tilde{p}_{i} z^{f\left(s_{i}, r\left(t_{k}\right), g_{i}\right)}\right) .
\end{aligned}
$$

To take the effects of multiple loading application on component UGF into account, the component UGF can be modified via (14) as follows:

$$
\begin{gathered}
U_{\max }\left(t_{k}\right)=\sum_{j=1}^{n_{2}} \widetilde{p}_{j}\left\{\bigotimes_{f_{1}}\left[U_{\widetilde{s} \max }, H\left(t_{k}\right)\right]\right\} \\
=\sum_{j=1}^{n_{2}} \widetilde{p}_{j}\left(\sum_{i_{1}=1}^{n_{1}} \sum_{i_{2}=1}^{n_{1}} \cdots \sum_{i_{n}=1}^{n_{1}} \prod_{j=1}^{n} \widetilde{p}_{i_{j}}\right. \\
\left.\cdot z^{f\left(\max \left(\widetilde{s}_{i_{1}}, \widetilde{s}_{i_{2}}, \ldots, \widetilde{s}_{i_{n}}\right), r\left(t_{k}\right), g_{i}\right)}\right) .
\end{gathered}
$$

For computational convenience, conventional timedependent reliability models of components with respect to load and strength always derive the reliability via the interference between load and strength at each time interval by using both the distribution function or membership function of load and that of strength as shown in Figure 3. When using the membership function of strength state probability at each time interval for component UGF calculation, strength degradation dependence (SDD) could be neglected, which might result in large calculation error in reliability. In this case, the component UGF can be derived by

$$
U\left(t_{k}\right)=\bigotimes_{f_{1}}\left(S, H\left(t_{k}\right)\right)=\sum_{i=1}^{n_{1}} \sum_{j=1}^{n_{2}} \widetilde{p}_{i} \tilde{p}_{j} z^{f\left(s_{i}, r\left(t_{k}\right), g_{i}\right)} .
$$

\section{System UGF and System Reliability}

Components UGFs in different situations are derived in the above section; they are the basis for system UGF and system reliability estimation. The fuzzy reliability of various systems with binary states, such as the series system, the parallel system, and the voting system, has been studied intensively. Nevertheless, fuzzy reliability models of multistate systems need further investigation. In this section, the fuzzy system UGF and system reliability models will be constructed.

4.1. UGF of Independent System. For series system and parallel system, assuming that the working states values of the two components are $g_{1}, g_{2}$, then the relationship between them can be expressed as [6]

$$
\begin{aligned}
& f_{\text {par }}\left(g_{1}, g_{2}\right)=\max \left(g_{1}, g_{2}\right), \\
& f_{\text {ser }}\left(g_{1}, g_{2}\right)=\min \left(g_{1}, g_{2}\right) .
\end{aligned}
$$




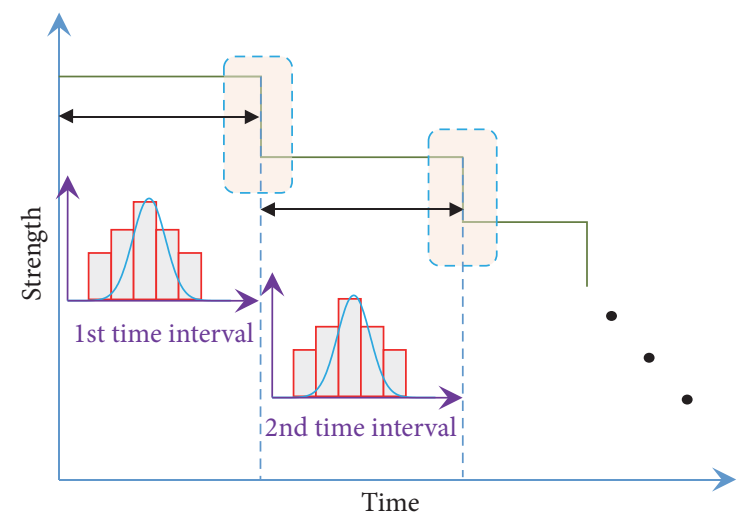

FIGURE 3: Strength state probability in different time intervals.

Therefore, the fuzzy UGF of the series system and the parallel system can be expressed as

$$
\begin{aligned}
\widetilde{U}_{\text {ser }} & =\bigotimes_{\min }\left(\widetilde{U}_{1}, \widetilde{U}_{2}, \ldots, \widetilde{U}_{n}\right) \\
& =\sum_{j_{1}=1}^{m_{1}} \sum_{j_{2}=1}^{m_{2}} \ldots \sum_{j_{n}=1}^{m_{n}} \prod_{i=1}^{n} \widetilde{p}_{i j_{i}} z^{\min \left(\widetilde{g}_{1 j_{1}}, \ldots, \widetilde{g}_{n j_{n}}\right)}, \\
\widetilde{U}_{\mathrm{par}} & =\bigotimes_{\max }\left(\widetilde{U}_{1}, \widetilde{U}_{2}, \ldots, \widetilde{U}_{n}\right) \\
& =\sum_{j_{1}=1}^{m_{1}} \sum_{j_{2}=1}^{m_{2}} \ldots \sum_{j_{n}=1}^{m_{n}} \prod_{i=1}^{n} \widetilde{p}_{i j_{i}} z^{\max \left(\widetilde{g}_{j_{1}}, \ldots, \widetilde{g}_{j_{n}}\right)} .
\end{aligned}
$$

When considering the strength degradation, the system UFG can be further written as

$$
\begin{aligned}
& \widetilde{U}_{\text {ser }}\left(t_{k}\right)=\bigotimes_{\min }\left(\widetilde{U}_{1}\left(t_{k}\right), \widetilde{U}_{2}\left(t_{k}\right), \ldots, \widetilde{U}_{n}\left(t_{k}\right)\right) \\
& =\sum_{j_{1}=1}^{m_{1}} \sum_{j_{2}=1}^{m_{2}} \cdots \sum_{j_{n}=1}^{m_{n}} \prod_{i=1}^{n} \widetilde{p}_{i j_{i}}\left(t_{k}\right) z^{\min \left(\widetilde{g}_{j_{1}}\left(t_{k}\right), \ldots, \widetilde{g}_{n j n}\left(t_{k}\right)\right)}, \\
& \widetilde{U}_{\text {par }}\left(t_{k}\right)=\bigotimes_{\max }\left(\widetilde{U}_{1}\left(t_{k}\right), \widetilde{U}_{2}\left(t_{k}\right), \ldots, \widetilde{U}_{n}\left(t_{k}\right)\right) \\
& =\sum_{j_{1}=1}^{m_{1}} \sum_{j_{2}=1}^{m_{2}} \cdots \sum_{j_{n}=1}^{m_{n}} \prod_{i=1}^{n} \widetilde{p}_{i j_{i}}\left(t_{k}\right) z^{\max \left(\widetilde{g}_{1 j_{1}}\left(t_{k}\right), \ldots, \widetilde{g}_{n j_{n}}\left(t_{k}\right)\right)} .
\end{aligned}
$$

4.2. UGF of Dependent System. When the components in a subsystem share a common load, the assumption that the components are mutually independent could lead to error in reliability calculation. To solve this problem, define decomposition operator $\underset{D}{\otimes}$ and inner product operator $\underset{\varphi \bullet}{\otimes}$ as follows, for two UGFs with the same number of expansion items

$$
\begin{aligned}
U_{1} & =\sum_{j=1}^{m} p_{1 j} z^{g_{1 j}}, \\
U_{2} & =\sum_{j=1}^{m} p_{2 j} z^{g_{2 j}} .
\end{aligned}
$$

The inner product operation is defined as

$$
U_{1} \underset{\varphi \bullet}{\otimes} U_{2}=\sum_{j=1}^{m} p_{1 j} p_{2 j} z^{\varphi\left(g_{1 j}, g_{2 j}\right)} .
$$

In addition, for any UGF, the decomposition can be performed via the decomposition operator as follows:

$$
U=\sum_{i=1}^{q} p_{i} z^{g_{i}}=\bar{U}_{1} \underset{D}{\otimes} \bar{U}_{2}
$$

where

$$
\begin{aligned}
& \bar{U}_{1}=\sum_{i=1}^{q} p_{i} z^{m} \quad(m=1) \\
& \bar{U}_{2}=\sum_{i=1}^{q} p_{i 2} z^{g_{i}} \quad\left(p_{i 2}=1\right) .
\end{aligned}
$$

$\bar{U}_{1}$, and $\bar{U}_{2}$ are termed as the first decomposition and the second decomposition, respectively. For a system composed of $n$ components sharing the same load with the first decomposition and the second decomposition of $\bar{S}_{\max 1}$ and $\bar{S}_{\max 2}$ in the $k$ th time interval, the system UGF $U_{s}$, considering strength degradation of components, can be obtained by (27) and (28) as follows:

$$
\begin{aligned}
\widetilde{U}_{i}\left(t_{k}\right)= & \bigotimes_{f}\left(\bar{S}_{\max 2}, H_{i}\left(t_{k}\right)\right) \\
= & \sum_{i=0}^{n_{1}} \sum_{r_{i}=0}^{n_{i}} p_{i} p_{r_{i}} z^{f\left(s_{i}, r_{i}\left(t_{k}\right), g_{S_{i}}\right)}, \\
U_{s}\left(t_{k}\right)= & \bar{S}_{\max 1} \\
& \underset{\times}{\otimes}\left(\underset{\varphi \cdot}{\otimes}\left(\widetilde{U}_{1}\left(t_{k}\right), \widetilde{U}_{2}\left(t_{k}\right), \ldots, \widetilde{U}_{n}\left(t_{k}\right)\right)\right) .
\end{aligned}
$$

In (28), the effects of common cause failure due to sharing the same load are taken into consideration.

4.3. System Reliability. The reliability of a multistate systems is defined as the probability that the system operational state is larger than the required demand. The reliability can be calculated by using the UGF by [6]:

$$
R(w)=P(G \geq w)=\sum_{G_{i} \geq w} p_{g_{i}} ;
$$

$G$ is the operating state value of the system, $w$ is the required performance index, and $p_{q_{i}}$ is the probability that $g_{i}$ is greater than or equal to $w$. When the UGF of the components is known, the UGFs of independent multistate systems and dependent multistate systems can be obtained by (22), (27), and (28). The reliability of independent multistate systems and dependent multistate systems can be obtained by (29). Assuming that the $j$ th operating state value is $\left(a_{j}, b_{j}, c_{j}\right)$ in systems and the performance index is $w$, then, the fuzzy 
UGF of the system for the performance index $w$ can be expressed as

$$
\widetilde{U}_{s w}=\sum_{j=1}^{n_{6}} \widetilde{p}_{w j}\left(p_{j} z^{1}+\left(1-p_{j}\right) z^{0}\right)
$$

where

$$
p_{j}= \begin{cases}1, & w<b_{j}, \\ 1-\frac{\left(w-b_{j}\right)^{2}}{\left(c_{j}-b_{j}\right)\left(a_{j}-b_{j}\right)}, & b_{j} \leq w<a_{j}, \\ \frac{\left(c_{j}-w\right)^{2}}{\left(c_{j}-b_{j}\right)\left(c_{j}-a_{j}\right)}, & a_{j}<w \leq c_{j}, \\ 0, & w>c_{j} .\end{cases}
$$

The fuzzy reliability of multistate system can be expressed as

$$
R=\frac{d \widetilde{U}_{s w}}{d z} .
$$

The index of dynamic reliability can be divided into transient reliability and reliability within a specified time duration. Transient reliability analysis is concerned with the reliability at a specified moment or a specified time interval. Reliability within a specified time duration is concerned with the possibility that a system does not fail at any time duration. In this paper, when considering strength degradation, the system reliability calculated by the proposed models belongs to the reliability within a specified time duration. The system reliability within $t$, which is composed of $N$ time interval, can be given by

$$
R(t)=\prod_{j=1}^{N} R_{j}\left(t_{j}, w\right),
$$

where $R_{j}\left(t_{j}, w\right)$ is the reliability in the $j$ th time interval.

\section{Numerical Examples}

Considering a series-parallel system as shown in Figure 4, the parallel subsystem 1 composed of Component 1 and Component 2 is connected in series with the parallel subsystem 2 composed of Component 3 and Component 4. Component 1 and Component 2 are subjected to the same loading and Component 3 and Component 4 share the same load. The fuzzy UGF of the load and that of the component strength are summarized as follows:

$$
\begin{aligned}
\widetilde{S}_{1}= & (0.02,0.01,0.03) z^{6}+(0.03,0.01,0.05) z^{5.5} \\
& +(0.05,0.03,0.07) z^{5}+0.1 z^{4.5} \\
& +(0.2,0.18,0.22) z^{4}+0.4 z^{3}+0.2 z^{2}, \\
\widetilde{S}_{2}= & (0.02,0.01,0.03) z^{6}+(0.02,0.01,0.03) z^{5.5} \\
& +(0.06,0.05,0.07) z^{5}+0.2 z^{4.5} \\
& +(0.3,0.28,0.32) z^{4}+0.3 z^{3}+0.1 z^{2},
\end{aligned}
$$
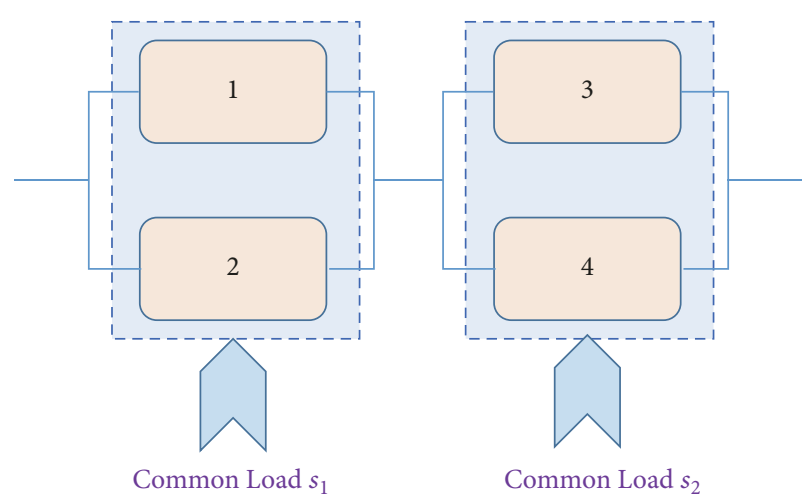

Figure 4: System structure.

$$
\begin{aligned}
H_{1}= & 0.1 z^{9}+0.2 z^{8.5}+0.1 z^{8}+0.1 z^{7.5}+0.1 z^{7} \\
& +0.1 z^{6.5}+0.2 z^{6}+0.1 z^{5}, \\
H_{2}= & 0.1 z^{9}+0.2 z^{8.5}+0.1 z^{8}+0.1 z^{7.5}+0.1 z^{7} \\
& +0.1 z^{6.5}+0.2 z^{6}+0.1 z^{5}, \\
H_{3}= & 0.1 z^{9}+0.1 z^{8.5}+0.1 z^{8}+0.1 z^{7.5}+0.1 z^{7} \\
& +0.1 z^{6.5}+0.25 z^{6}+0.15 z^{5}, \\
H_{4}= & 0.1 z^{9}+0.1 z^{8.5}+0.1 z^{8}+0.1 z^{7.5}+0.1 z^{7} \\
& +0.1 z^{6.5}+0.25 z^{6}+0.15 z^{5} .
\end{aligned}
$$

The working states corresponding to $s_{1}, s_{2}$ are $[1,2,3,4,5$, $6,7]$.

When the components in the systems are assumed to be mutually statistically independent and be subjected to the load independently, the fuzzy reliability of the system without the effects of multiple load application taken into consideration under the performance requirements of $w=3, w=4$, and $w=5$, respectively, can be seen in Figure 5. In addition, the fuzzy reliability of the dependent system considering the effects of common load resources under the required performance of $w=3, w=4$, and $w=5$, respectively, is plotted in Figure 6.

From Figures 5 and 6, it can be learned that the system reliability decreases when considering the failure dependence of components caused by the common load resources. Furthermore, the reliability under high performance requirement decreases faster than the reliability under low performance requirement. There are two subsystems in the system, in which the components share the same load. Thus, for the system in parallel configuration, the ability to resist the risk of failure could be largely weakened due to the existence of common load. Moreover, the widths of the fuzzy system reliability of the dependent system under different performance requirements are larger than that of the independent system, which means that the fuzziness of reliability is more obvious for dependent system. In this case, for dependent systems, there needs to be more efforts to obtain more information 


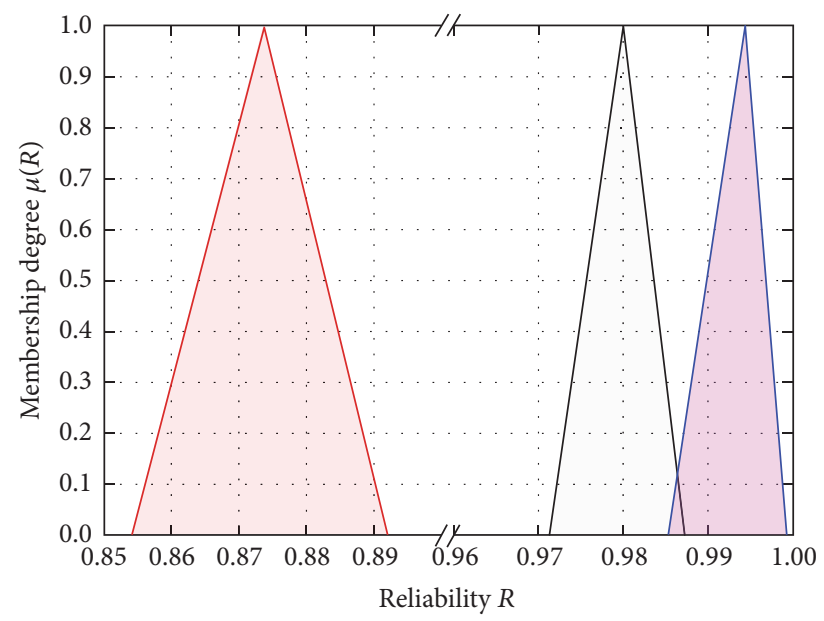

$w=5$

$w=4$

$w=3$

Figure 5: Fuzzy reliability of independent system.

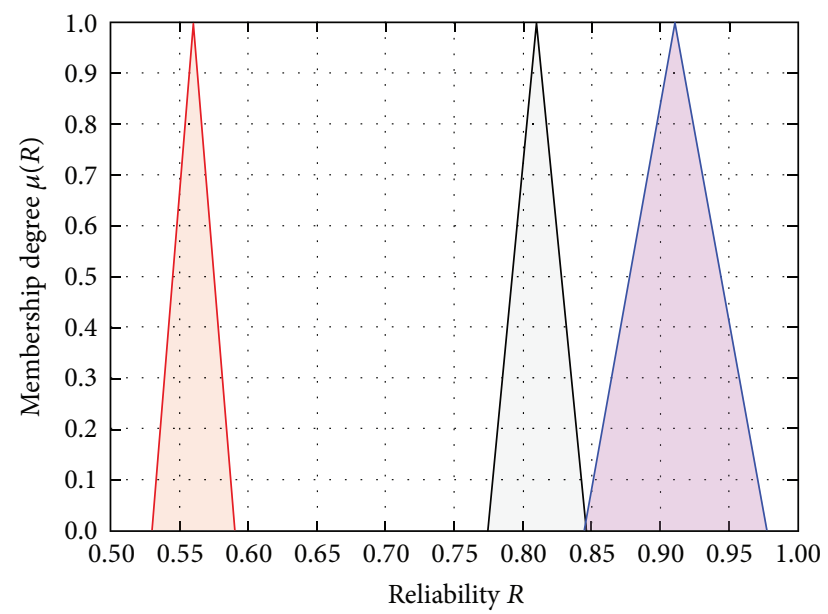

$w=5$

$w=4$

$w=3$

FIGURE 6: Fuzzy reliability of dependent system.

in order to decease the fuzziness of reliability and achieve an accurate assessment of system reliability.

In addition, when considering the effects of multiple load applications (in this numerical example, the two loads apply for six times, resp.), the fuzzy reliability of the dependent system under the performance requirements of $w=3, w=4$, and $w=5$, respectively, is shown in Figure 7. As can be seen from Figure 7, the system reliability under high performance requirement is obviously reduced when considering the effect of load times. This is because the failure probability of the reliability increases with the increase in the load application times. Meanwhile, the fuzziness of system reliability under low performance requirements is more obvious than that under high performance requirements.

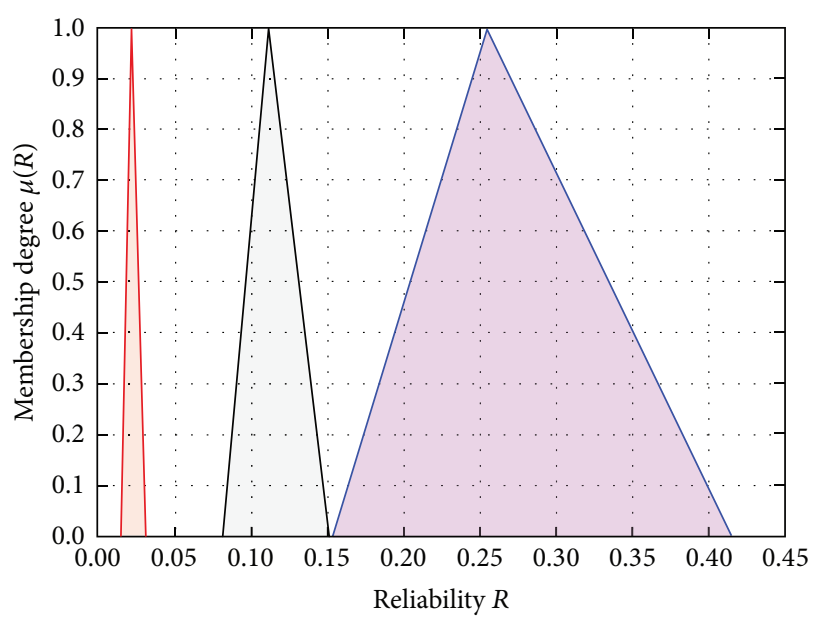

$w=5$

$w=4$

$w=3$

Figure 7: Fuzzy reliability of dependent system considering the effects of multiple load applications.

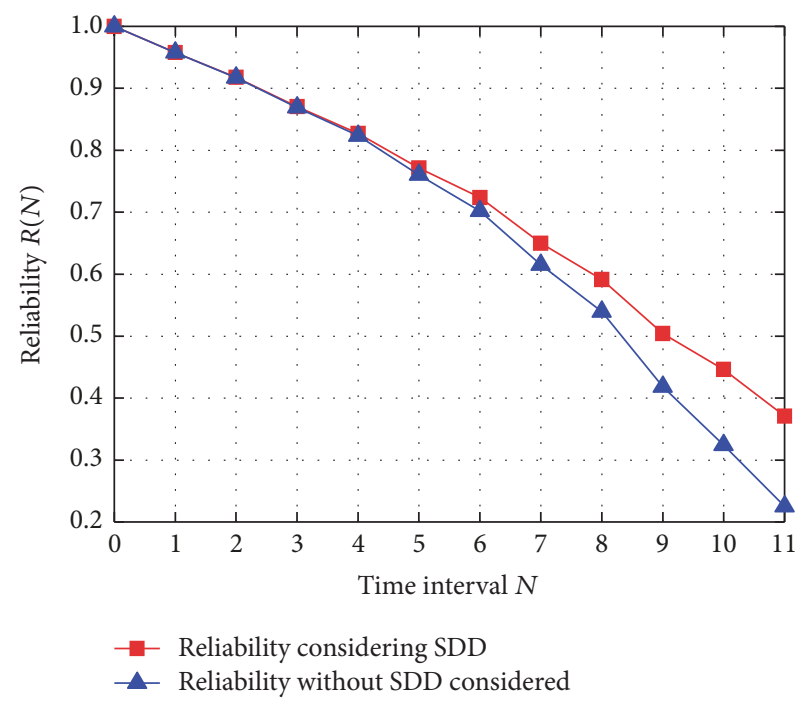

FIGURE 8: Fuzzy reliability of dependent system considering strength degradation.

To analyze the fuzzy system reliability with strength degradation, the initial strength states are assumed to be $r=$ $[9,8.5,8,7.5,7,6.5,6,5]$. The strength states in the $N$ th time intervals are expressed by the degradation law as follows:

$$
r(N)=r-(0.25 * N) .
$$

For the convenience of analysis, the center points of the fuzzy system reliability in different time intervals are shown in Figure 8.

From Figure 8, it can be seen that the system reliability decreases with the strength degradation of components. In practice, strength degradation takes place frequently in various technological systems. Conventional static reliability 
models cannot be used to represent the dynamic characteristics of fuzzy system reliability. Therefore, the proposed models provide an approach for dynamic reliability analysis of multistate systems. In addition, SDD has great influences on system reliability. In the situation where SDD is not taken into account, the system reliability could be seriously underestimated, which could lead to misleading for the risk analysis of multistate systems. Moreover, this error in reliability estimation increases with time.

\section{Conclusions}

Fuzzy reliability models of multistate systems are developed in this paper. In the proposed models, the parameters of load and strength are taken into account when deriving the system UGF and calculating the system reliability. By using the fuzzy load UGF and the fuzzy strength UGF, the proposed models can be used to address the problems of failure dependence and effects of multiple load applications in system reliability estimation. Besides, the strength degradation is considered in the proposed dynamic fuzzy reliability models of multistate systems. The dynamic reliability models can be used to represent the dynamic characteristics of system reliability. The results show that failure dependence and the effects of multiple load applications have significant impacts on system reliability, which considerably decreases system reliability and increases the fuzziness of system reliability under low performance requirements. In addition, strength degradation has great influences on system reliability. System reliability decreases with strength degradation of components. In the calculation of dynamic system reliability, SDD could result in large error. Therefore, attention should be paid to SDD in dynamic reliability assessment of multistate systems.

\section{Conflicts of Interest}

The authors declare that there are no conflicts of interest regarding the publication of this paper.

\section{Acknowledgments}

This work is supported by the National Natural Science Foundation of China (Grant no. 51505207), the Program for Liaoning Innovative Talents in University (Grant no. LR2017070), Liaoning Provincial Natural Science Foundation of China (Grant no. 2015020152), and the Open Foundation of Zhejiang Provincial Top Key Academic Discipline of Mechanical Engineering (Grant no. ZSTUME02A01).

\section{References}

[1] C.-M. Feng and C.-C. Chung, "Assessing the risks of airport airside through the fuzzy logic-based failure modes, effect, and criticality analysis," Mathematical Problems in Engineering, vol. 2013, Article ID 239523, pp. 289-307, 2013.

[2] I. Kurkova and K. Raschel, "New steps in walks with small steps in the quarter plane: series expressions for the generating functions," Annals of Combinatorics, vol. 19, no. 3, pp. 461-511, 2015.
[3] B. Dherin, "The universal generating function of analytical Poisson structures," Letters in Mathematical Physics, vol. 75, no. 2, pp. 129-149, 2006.

[4] T. Jin, M. Zhou, and G. Li, "Universal generating function based probabilistic production simulation for wind power integrated power systems," Journal of Modern Power Systems and Clean Energy, vol. 5, no. 1, pp. 134-141, 2017.

[5] M. A. Farsi, "Develop a new method to reliability determination of a solar array mechanism via universal generating function," Journal of Mechanical Science and Technology, vol. 31, no. 4, pp. 1763-1771, 2017.

[6] G. Levitin, The universal generating function in reliability analysis and optimization, Springer, Berlin, Germany, 2005.

[7] G. Levitin, A. Lisnianski, and D. Elmakis, "Structure optimization of power system with different redundant elements," Electric Power Systems Research, vol. 43, no. 1, pp. 19-27, 1997.

[8] A. Lisnianski, G. Levitin, H. Ben-Haim, and D. Elmakis, "Power system structure optimization subject to reliability constraints," Electric Power Systems Research, vol. 39, no. 2, pp. 145-152, 1996.

[9] Y. Ding and A. Lisnianski, "Fuzzy universal generating functions for multi-state system reliability assessment," Fuzzy Sets and Systems, vol. 159, no. 3, pp. 307-324, 2008.

[10] Z.-W. An, H.-Z. Huang, and Y. Liu, "A discrete stress-strength interference model based on universal generating function," Reliability Engineering \& System Safety, vol. 93, no. 10, pp. 14851490, 2008.

[11] Z. Jinyu, X. Liyang, and W. Xuemin, "Common cause failure analysis and reliability model for multi-state system," in Journal of Mechanical Engineering, vol. 41, pp. 66-67, 6 edition, 2005.

[12] C. Wang, Z. Qiu, M. Xu, and H. Qiu, "Novel fuzzy reliability analysis for heat transfer system based on interval ranking method," International Journal of Thermal Sciences, vol. 116, pp. 234-241, 2017.

[13] A. K. Aggarwal, S. Kumar, and V. Singh, "Mathematical modeling and fuzzy availability analysis for serial processes in the crystallization system of a sugar plant," Journal of Industrial Engineering International, vol. 13, no. 1, pp. 47-58, 2017.

[14] L. Bing, Z. Meilin, and X. Kai, "Practical engineering method for fuzzy reliability analysis of mechanical structures," Reliability Engineering \& System Safety, vol. 67, no. 3, pp. 311-315, 2000.

[15] Li. Yanjie and G. He, "Fuzzy reliability of series system," Fuzzy systems and Mathematics, vol. 3, pp. 38-45, 1989.

[16] L. Yanjie and G. He, "Fuzzy reliability of series-parallel system," in System Engineering Theory and Practice, vol. 3, pp. 11-14, 2 edition, 1990.

[17] F. Biondini, F. Bontempi, and P. G. Malerba, "Fuzzy reliability analysis of concrete structures," Computers \& Structures, vol. 82, no. 13-14, pp. 1033-1052, 2004.

[18] Y. Massim, A. Zeblah, M. Benguediab, A. Ghouraf, and R. Meziane, "Reliability evaluation of electrical power systems including multi-state considerations," Electrical Engineering, vol. 88, no. 2, pp. 109-116, 2006.

[19] A. Maturo and A. G. Ventre, "Fuzzy Numbers and Consensus," in Soft Computing Applications for Group Decision-making and Consensus Modeling, vol. 357 of Studies in Fuzziness and Soft Computing, pp. 441-450, Springer International Publishing, Berlin, Germany, 2018. 


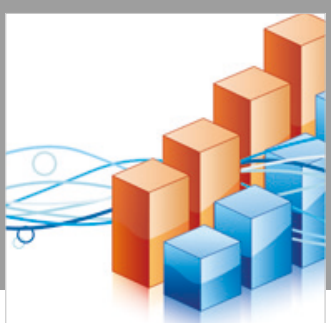

Advances in

Operations Research

\section{-n-m}
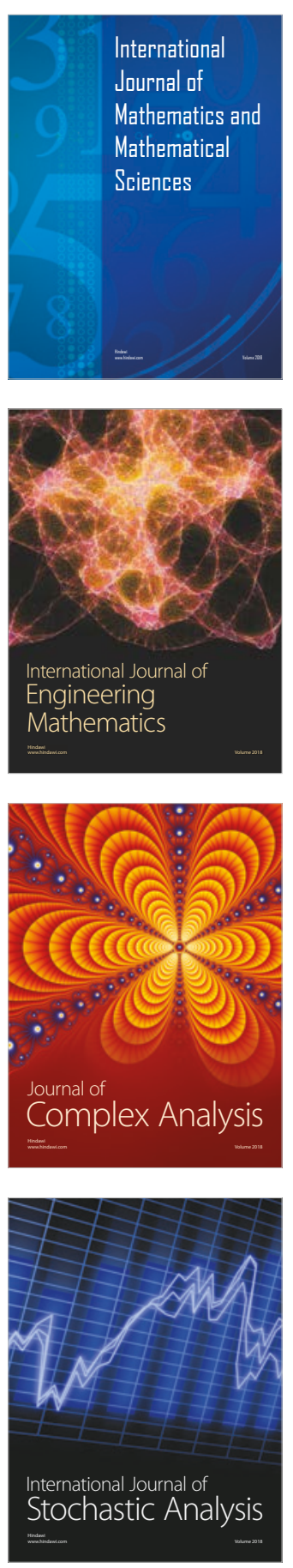
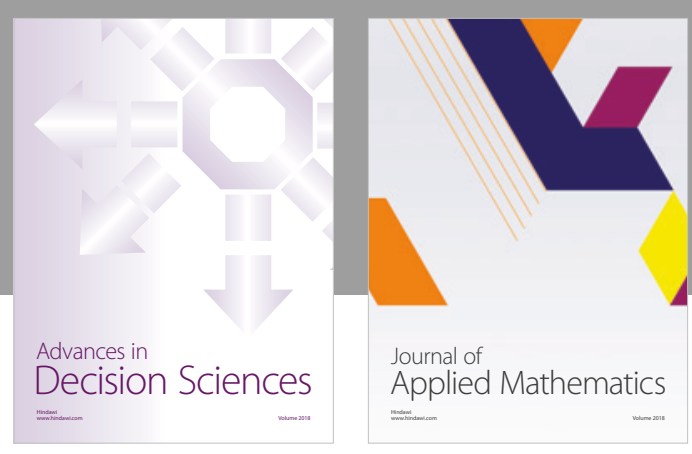

Journal of

Applied Mathematics
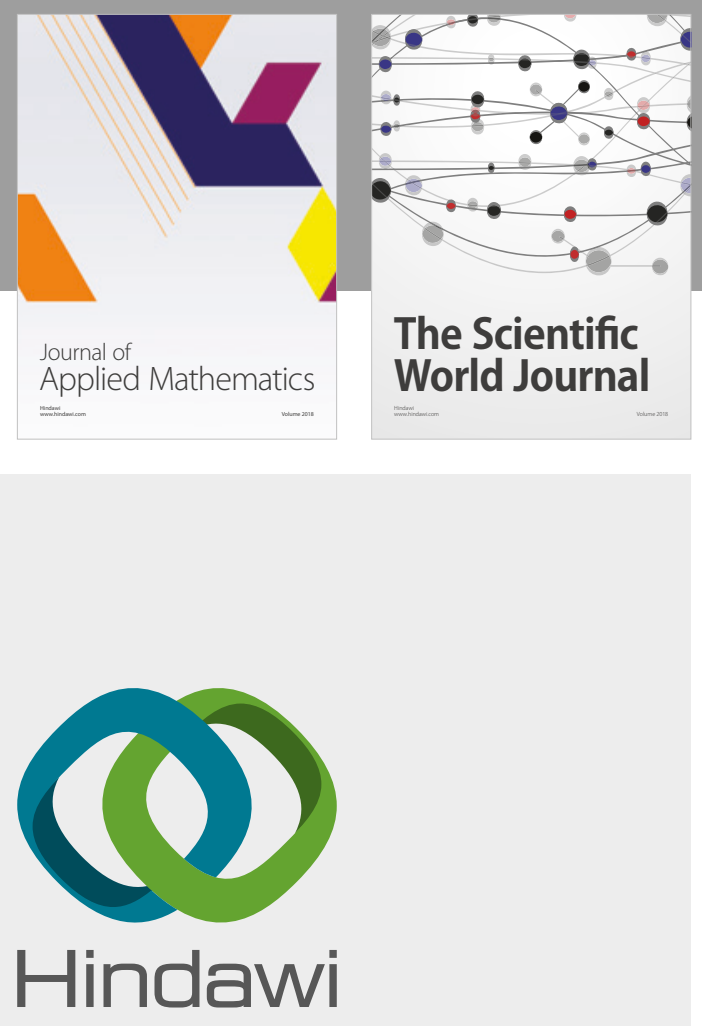

Submit your manuscripts at

www.hindawi.com

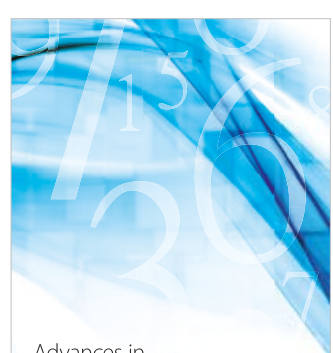

Advances in
Numerical Analysis
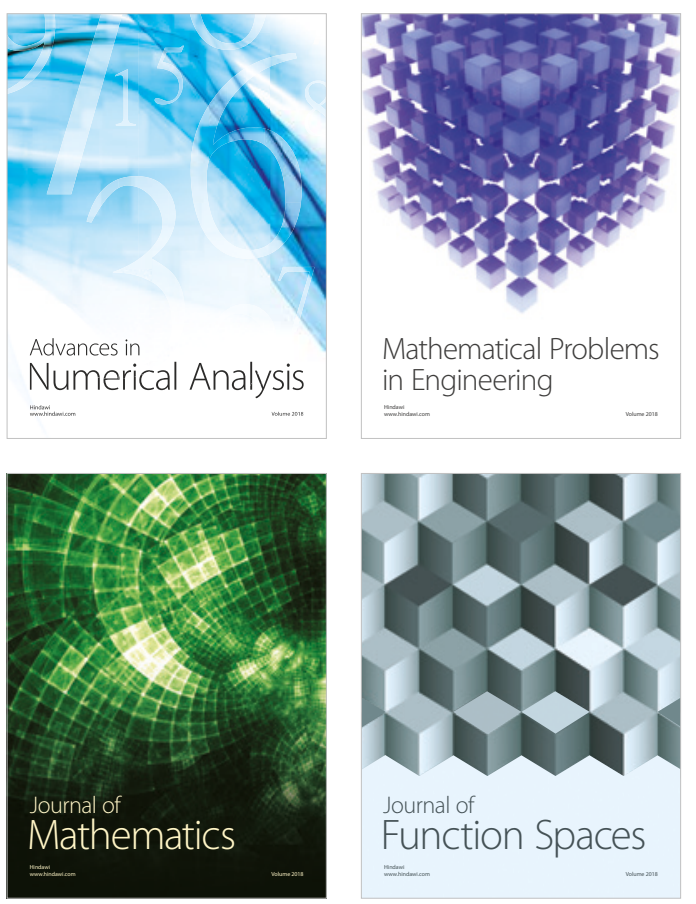

Mathematical Problems in Engineering

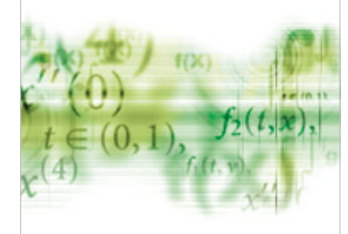

International Journal of

Differential Equations

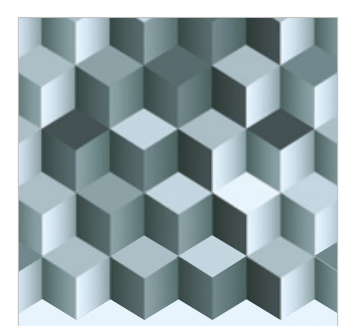

Journal of

Function Spaces

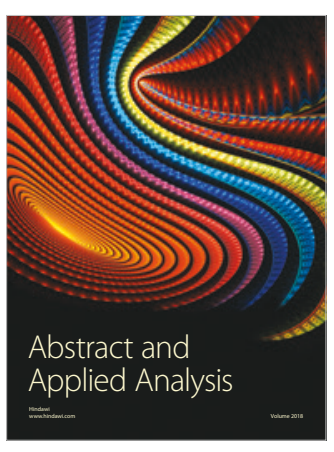

The Scientific

World Journal

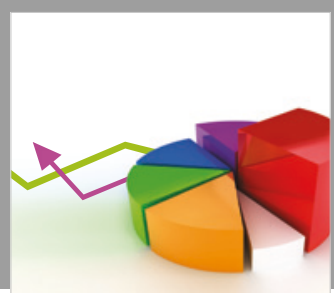

Journal of

Probability and Statistics
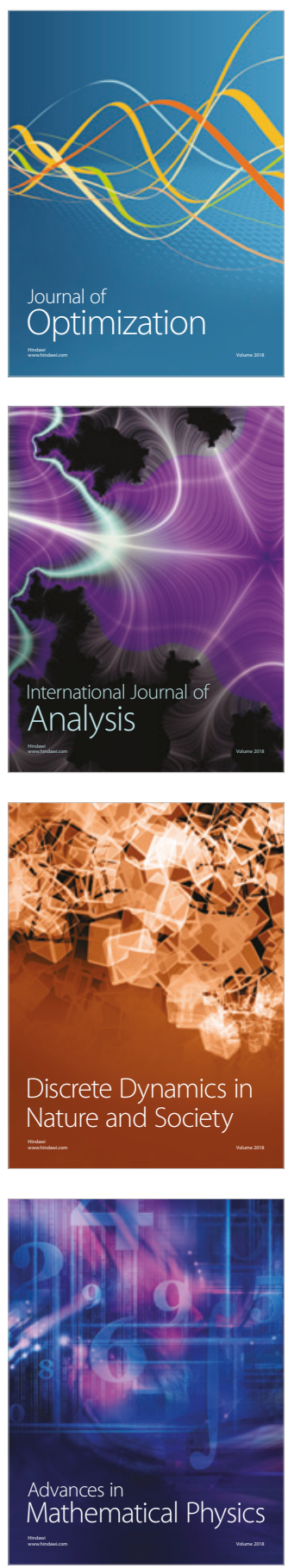